\title{
The Effects of Primary Elevation of Cerebral Venous Pressure on Cerebral Hemodynamics and Intracranial Pressure
}

\author{
J. E. Raisis, M.D., G. W. Kindt, M.D., J. E. McGillicuddy, M.D., \\ and S. L. Giannotta, M.D. \\ Section of Neurosurgery, University of Michigan Medical Center, \\ Ann Arbor, Michigan 48109
}

Submitted for publication June 29, 1978

\section{INTRODUCTION}

The dynamics of an elevated cerebral venous pressure and its effect on cerebrospinal fluid (CSF) pressure have been studied in considerable detail in the past $[1,4$, $11,14,15]$. However, controversy still exists over the relationships between these pressures. As early as 1887 Falkenheim and Naunyn [5] observed that venous occlusion at the right atrium resulted in a rise in intracranial pressure (ICP). Queckenstedt [13] in 1916 demonstrated the clinical importance of the relationship between cerebral venous pressure and ICP. The results of the previous studies have varied; some showing ICP consistently greater than cerebral venous pressure and others showing the reverse to be true.

More recently, investigators have concerned themselves principally with the effects of primary elevation of ICP on the sagittal sinus wedge pressure and cerebral blood flow (CBF) [ 4, 10, 12, 14]. There have been few reports concerning primary elevation of cerebral venous pressure and its effect upon CBF and ICP. Also, no reports are available on the effects of marked (over $30 \mathrm{~mm} \mathrm{Hg}$ ) elevations of cerebral venous pressure. For this report, a series of experiments were performed on the primate to determine the effect on ICP of a primary elevation of cerebral venous pressure. During each experiment the cerebral venous pressure was raised slowly to the systemic arterial pressure (SAP) while the effect on
CBF was investigated. The pial vessels were observed directly through a watertight window as part of the experiment.

\section{METHODS}

Tracheostomies were performed on eight Rhesus monkeys weighing 5 to $7 \mathrm{~kg}$, that had been anesthetized with ketamine. The animals were ventilated with a constant volume respirator to maintain stable blood gases. Carotid blood flow was monitored by placing an electromagnetic flow probe around one common carotid artery after the ipsilateral external carotid artery had been ligated. When total CBF measurements were desired, the opposite carotid and both vertebral arteries were temporarily occluded. The ICP was monitored by the method of Gosch and Kindt [8].

A large bore polyethylene catheter was inserted into the inferior vena cava through one femoral vein. A variable speed roller pump was used to pump blood in a retrograde direction from the inferior vena cava into one jugular vein at the base of the skull. A catheter was placed in the opposite jugular vein to the base of the skull to monitor jugular bulb wedge pressure. This pressure was taken as a reflection of the cerebral venous pressure. The jugular bulb wedge pressure and sagittal sinus pressure were compared in this preparation. Measurements obtained demonstrated that these pressures were nearly equal quantitatively, and that pulsations were synchronous. A 
small craniectomy was performed and a water-tight lexan window was inserted with the inner aspect of the window fitting smoothly with the inner table of the skull. Using this window we were able to directly observe and photograph pial vessels with an operating microscope at $40 \times$ magnification. An additional trephine was placed on the opposite side of the skull so that ICP could be temporarily elevated by infusing artificial CSF into the subdural space. Pulse rate and SAP were continuously monitored throughout the experiment.

The ICP and CBF, as reflected by internal carotid flow, were monitored continuously while the cerebral venous pressure was raised. Both the qualitative and quantitative relationships between these entities were examined in detail. Control studies at normal cerebral venous pressure were made during which the ICP was primarily elevated by infusing artificial CSF into the subdural space. Steady-state conditions were not usually achieved during the primary elevations of cerebral venous pressure or ICP except at the beginning of the experimental runs. Brain swelling and decompensation of the preparation occurred when cerebral venous pressure or ICP were maintained at an elevated level for any long period. The data obtained was thus in the form of dynamic curves of changes in CBF or ICP as the cerebral venous pressure was elevated. Curves that were representative of the changes observed during the multiple experimental runs were reproduced (Figs. 1 and 2).

The pial vessels were observed directly through the cranial window. The reactions of the pial vessels to an elevated cerebral venous pressure, as well as to an elevated ICP, were photographed and compared. Correlation of the observed vascular changes was made with the measured CBF.

\section{RESULTS}

Pumping the venous blood in a cephalad direction proved to be a simple method of adjusting and monitoring the cerebral ve-

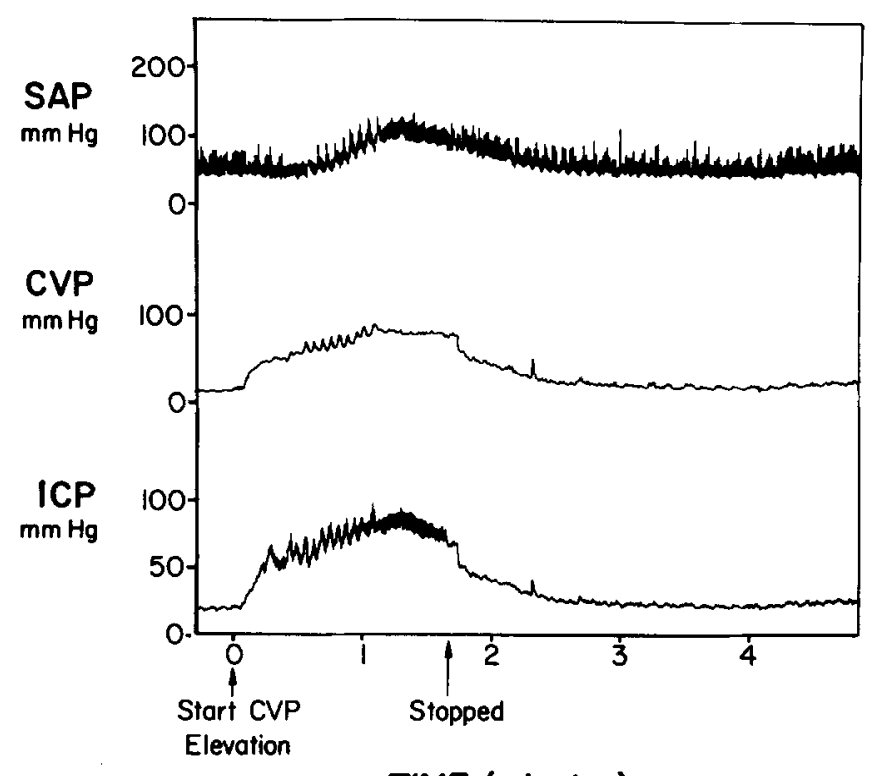

TIME (minutes)

FIG. 1. An example of the acute effects of a primary elevation of cerebral venous pressure on ICP and SAP. Note the Cushing response produced by the marked elevations in cerebral venous pressure and ICP. 
nous pressure at any desired level. We saw no evidence of compromise in cardiovascular function even when the CVP was raised to arterial pressure levels. A high cerebral venous pressure and ICP were maintained for only 2 - to 3 -min intervals to avoid brain damage and decompensation.

The data from our experiments demonstrate the effect of an elevated cerebral venous pressure on the cerebral circulation and ICP. The relationships between cerebral venous pressure, ICP, and SAP are illustrated in Fig. 1. Elevation of cerebral venous pressure triggered a prompt and simultaneous rise in ICP. Pulsations of both pressures appeared synchronous and relatively equal in intensity. During this part of the experiment, ICP fluctuated but remained within $5 \%$ of the venous pressure level. When cerebral venous pressure was elevated to higher levels, approaching SAP, a Cushing response was initiated with associated elevation in SAP and slowing of the pulse [2].

The relation between cerebral venous pressure and CBF is demonstrated in Fig. 2. When cerebral venous pressure was elevated there was again a simultaneous increase in ICP. With an elevation in cerebral venous pressure to $75 \mathrm{~mm} \mathrm{Hg}, \mathrm{CBF}$ remained constant; however, with a more pronounced elevation in cerebral venous pressure approaching SAP, there was a precipitous drop in CBF. Reduction of cerebral venous pressure to control levels produced a rapid rise in $\mathrm{CBF}$, initially exceeding control values (see Fig. 2).

The pial vessels were directly observed while cerebral venous pressure was elevated using the method described. The response to various levels of cerebral venous pressure is demonstrated in Fig. 3B. With moderate elevation $(40-60 \mathrm{~mm} \mathrm{Hg})$ in cerebral venous pressure, pial vessels rapidly dilated. At higher venous pressures (above $80 \mathrm{~mm} \mathrm{Hg}$ ), there was flattening of the gyri against the lexan window. Pial vessels within the sulci remained dilated. Close observation of these vessels revealed that the

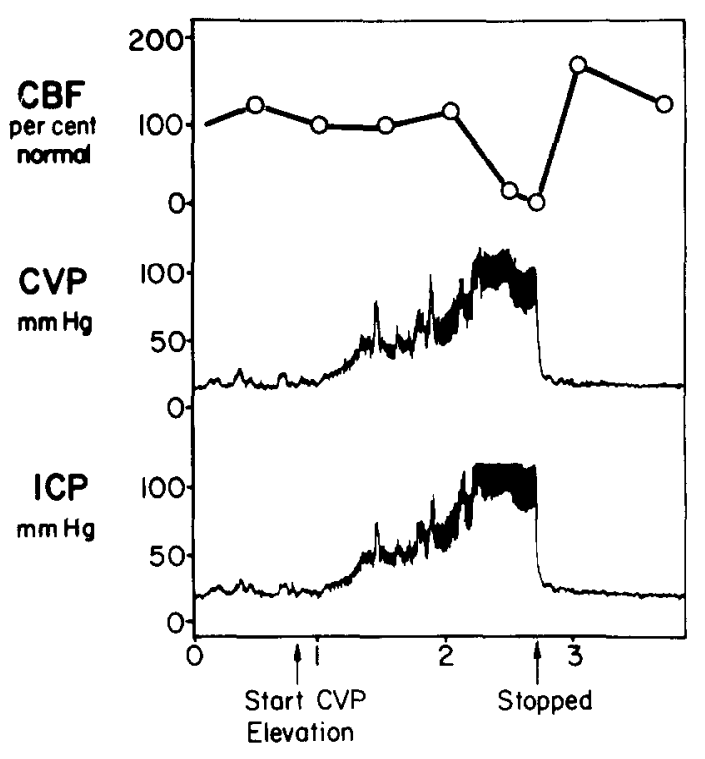

TIME (minutes)

FIG. 2. The effect of elevating cerebral venous pressure on ICP and CBF. The ICP changes directly with the cerebral venous pressure. CBF is at first maintained but then falls at high levels of cerebral venous pressure.

blood flow within them had stopped. On a few occasions, reversal of the flow was observed.

The pial vessels were also observed at various levels of primary elevation in ICP, and the response was compared with that seen when cerebral venous pressure was elevated (Fig. 3A). With elevation from 40 to $60 \mathrm{~mm} \mathrm{Hg}$ vasodilatation occurred. With more pronounced elevation of ICP there was flattening of the gyri, and cessation of the blood flow in pial vessels. The response seen with primary elevation of the ICP was virtually the same as that seen with a primarily elevated cerebral venous pressure.

\section{DISCUSSION}

This experimental study emphasizes the importance of cerebral venous pressure and its influence on ICP and CBF. A detailed, quantitative study by Becht in 1920 demonstrated the relationship between cerebral venous pressure and ICP [1]. Based on his experimental studies he concluded that "increased venous pressure uncomplicated by 


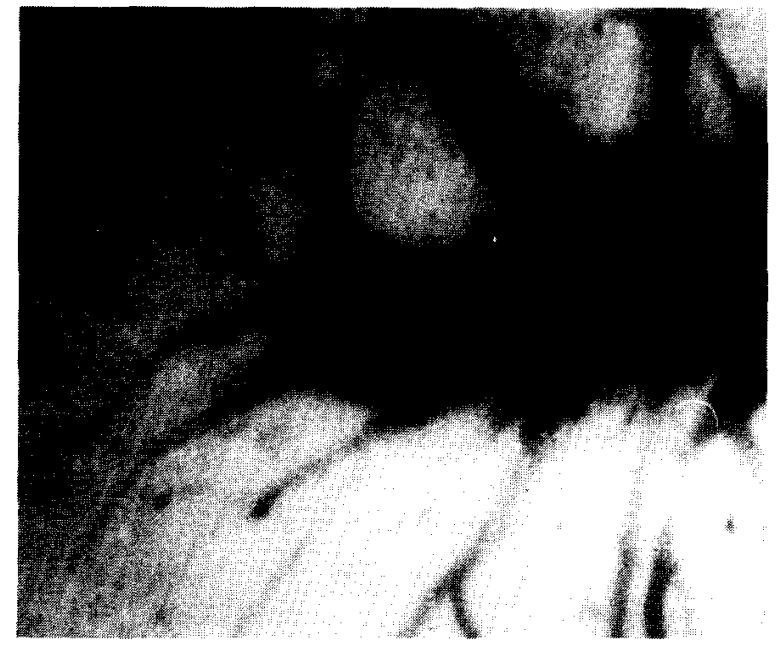



ฮี

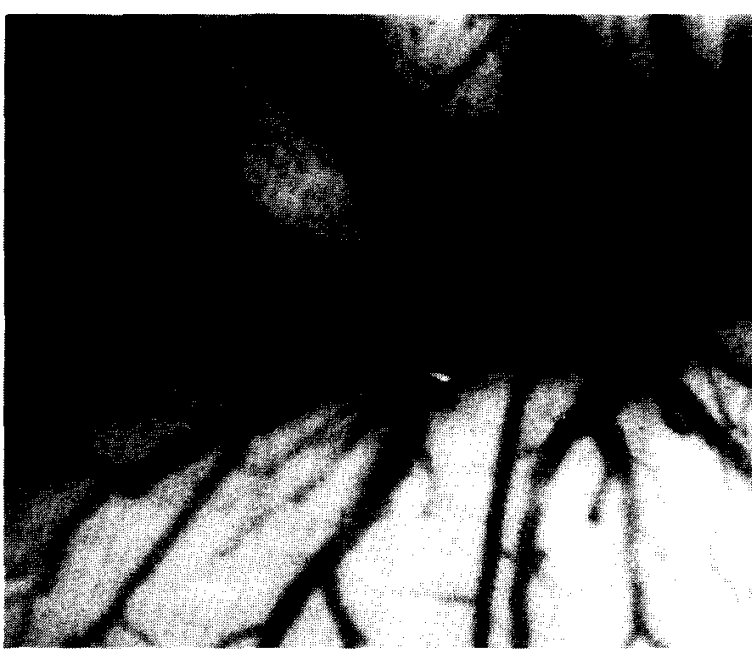

吾

언 풀

㝳

(1)

을

尔

政

ते

㤩

要

密

守

논

음

Z

牙

들

害旦

흔

늘

웜

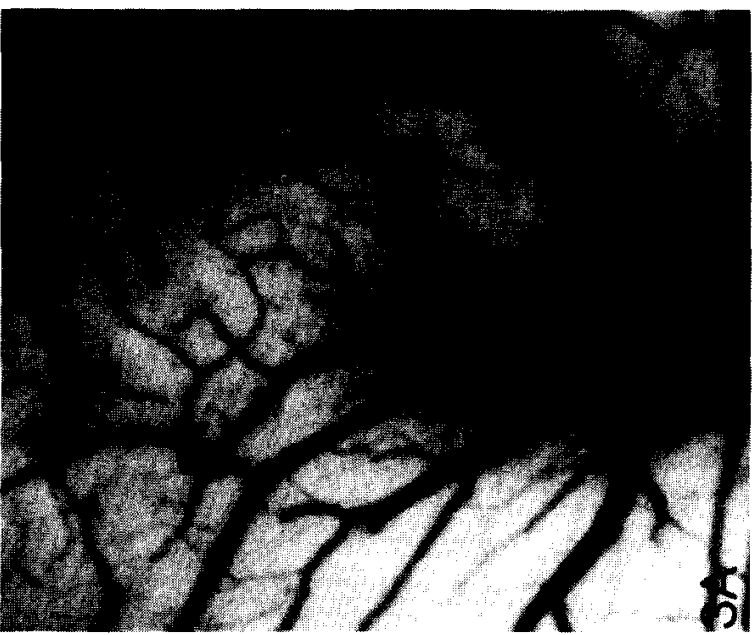

$\geq$ 롱

部

论

藏

鞄

造

记

赔

芩

ن

理 


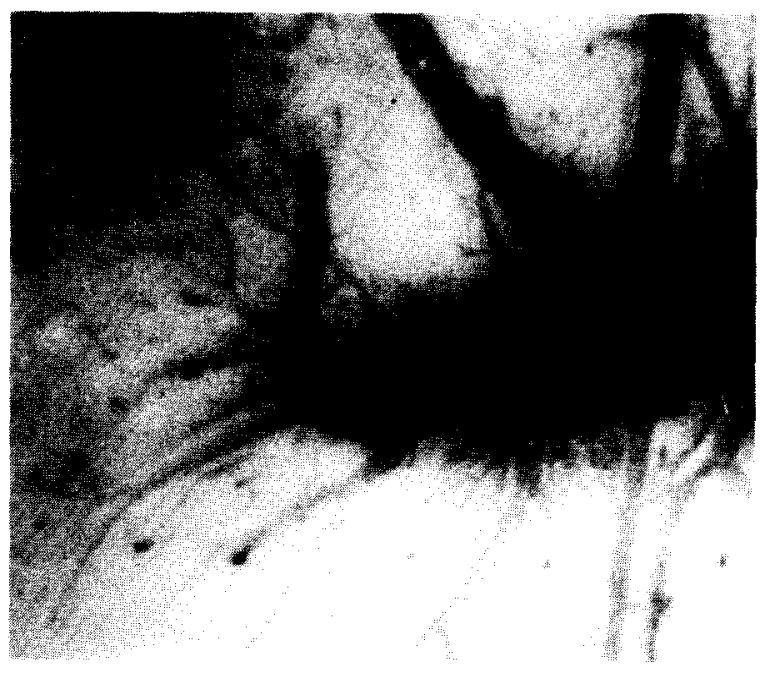

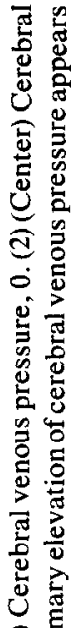

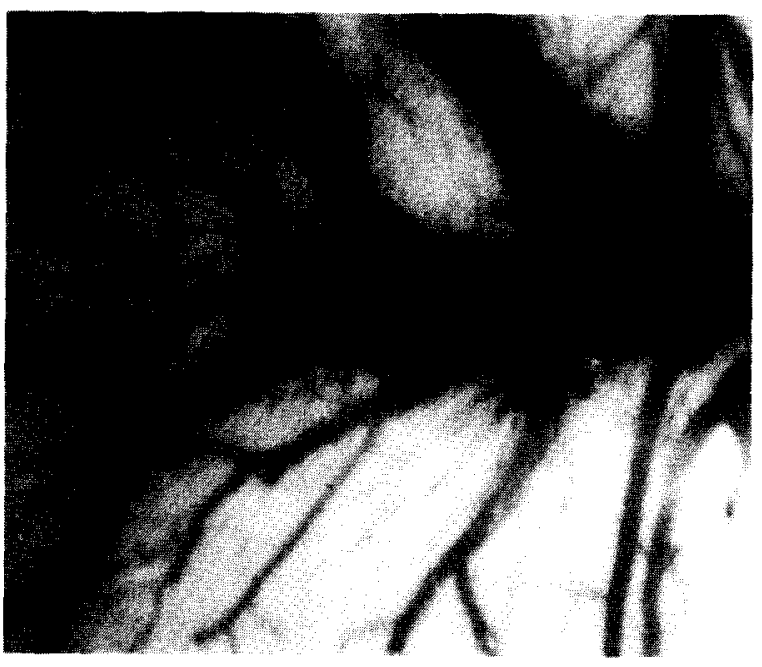

क्षे

尝

ฮัฐ

焉

窎

氶

空

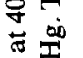

䆑主

递

응

$>$

渮

ธ气

究告

สํํำ

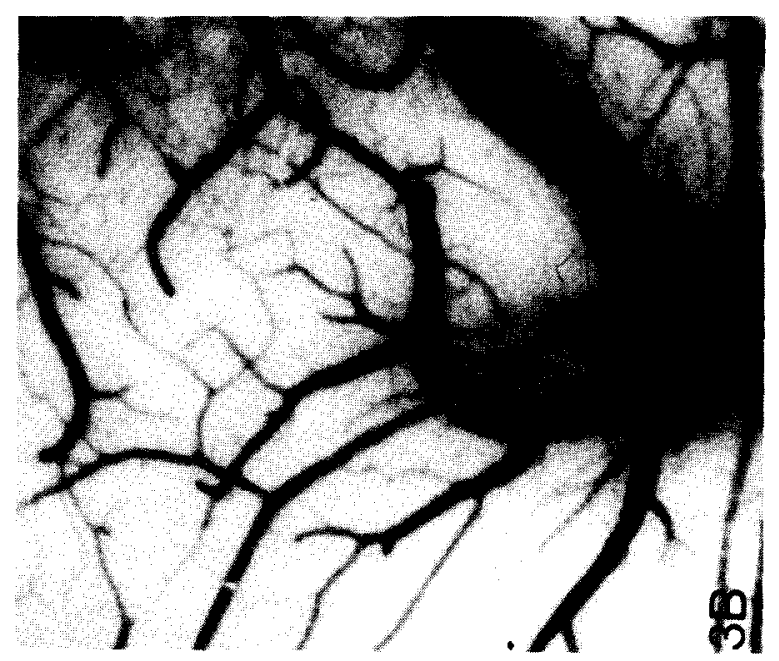

들

苍全.

도음

응

ग

要

ธํㅗㅇ

点

密客要

额

要

学容

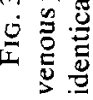


any marked changes in arterial pressure is accompanied by an increase in CSF pressure." Our experimental data (Fig. 1) agree with Becht [1] and others [7, 15] that there is a direct relationship between cerebral venous pressure and ICP, i.e., changes in cerebral venous pressure induce a similar change in ICP which is approximately equal in intensity.

The etiology for this direct relationship can be postulated. An accumulation of blood in the easily distensible cerebral venous system occurs with an elevated cerebral venous pressure. The venous dilatation is associated with an increased blood volume within the rigid cranial vault and produces a rise in the ICP. The arterial inflow is of such volume and pressure that these dynamic changes are almost instantaneous.

Ekström-Jodal [4] in a detailed monograph demonstrated the presence of autoregulation of CBF with elevated cerebral venous pressure. The cerebral venous pressure was not elevated to high levels with her model. Our observations (Fig. 2) demonstrate that with moderate elevations in cerebral venous pressure, the CBF remains constant. This steady flow suggests that CBF can autoregulate to moderate increases in cerebral venous pressure. More pronounced elevations in cerebral venous pressure lead to a sharp decrease in CBF. This decrease was likely caused by a reduction in cerebral perfusion pressure (CPP) below the level at which autoregulation can compensate. When cercbral venous pressure was reduced to control levels, there was a rapid rise in CBF. Initially this rise was above control values, suggesting reactive hyperemia.

There has becn continued interest in correlating the direct observation of pial vessels with suspected hemodynamic changes. Fog [6] directly observed the constriction of pial vessels during systemic hypertension in 1939. Wolf and Forbes [16] noted dilatation of pial vessels during gradual elevation of ICP. This vasodilatation is believed to be evidence of autoregulation of cerebral blood flow induced by raising ICP and the associated decrease in CPP $[9,17]$. Our observations of vasodilatation during elevated ICP were similar to those of Wolf and Forbes [16].

There have been few observations of the pial vessels during primary elevation of cerebral venous pressure [3]. We noted vasodilatation of pial vessels with an elevated cerebral venous pressure when compared with controls at normal cerebral venous pressure. This dilatation probably represented autoregulation of CBF to elevated cerebral venous pressure. More pronounced elevations in cerebral venous pressure resulted in cessation in blood flow. The observed response was virtually the same as that seen with gradual primary elevation of ICP.

The mechanism involved in autoregulation of CBF to an elevated cerebral venous pressure can be postulated. Ekström-Jodal [4] has suggested that autoregulation to an elevated cerebral venous pressure is due to a myogenic response produced by changes in transmural pressure. In our experiments a prompt rise in ICP was noted with all primary elevations of cerebral venous pressure. In this manner, a change in transmural pressure was produced in the process of raising cerebral venous pressure. Autoregulation to an elevated cerebral venous pressure may thus have occurred secondarily through a mechanism identical to that seen with a primarily elevated ICP [17]. Perhaps in other instances, such as with an open skull, an elevated cerebral venous pressure could affect the CPP and thus the CBF, without changing ICP.

Under normal circumstances, the cerebral venous pressure has little effect on cerebral hemodynamics. However, the cerebral venous pressure could be an important factor in certain clinical situations. Elevations in cerebral venous pressure have been shown to cause cerebral edema in the presence of damaged brain [3]. There are some patients who have borderline regional or total cerebral perfusion due to such abnormalities as arterial insufficiency, increased ICP, cerebral edema, or a combination of 
these. In such patients, elevation of cerebral venous pressure might decrease CPP enough to lower regional or total CBF to critical levels.

\section{ACKNOWLEDGMENTS}

This work was supported in part by the Michigan Heart Association Grant No. 341249 and the BegoleBrownell Neurosurgical Fund.

\section{REFERENCES}

1. Becht, F. Studies on the cerebrospinal fluid. Amer. J. Physiol. 51: 1, 1920.

2. Cushing, H. Concerning a definite regulatory mechanism of the vasomotor centre which controls blood pressure during cerebral compression. Bull. Johns Hopkins Hosp. 12: 290, 1901.

3. Cuypers, J., Matakas, F., and Potolicchio, S. J., Jr. Effect of central venous pressure on brain tissue pressure and brain volume. $J$. Neurosurg. 45: 89, 1976.

4. Ekström-Jodal, B. On the relationship between blood pressure and blood fiow in the canine brain with particular regard to the mechanism responsible for cerebral blood flow autoregulation. Acta Physiol. Scand. Suppl. 350: 1, 1970.

5. Falkenheim, H., and Naunyn, B. Ueber Hirndruck. Arch. Exp. Pathol. Pharmacol. 22: 261, 1887.

6. Fog, M. II, Reaction of pial arteries to increase in blood pressure. Arch. Neurol. Psychiatr. (Chicago) 41: 260, 1939.

7. Frazier, C. H., and Peet, M. M. Factors of influence in the origin and circulation of the cerebrospinal fluid. Amer. J. Physiol. 35: 268, 1914.

8. Gosch, H. H., and Kindt, G. W. Subdural monitoring of acute increased intracranial pressure. Surg. Forum 23: 405, 1972.

9. Langfitt, T. W. Increased intracranial pressure. Clin. Neurosurg. 16: 436, 1969.

10. Langfitt, T. W., Weinstein, J. D., Kassell, N. F., Gagliardi, L. J., and Shapiro, H. M. Compression of cerebral vessels by intracranial hypertension: 1, Dural sinus pressures. Acta Neurochir. 15: 212, 1966.

11. Meyerson, A., and Loman, J. Intracranial jugular venous pressure and its relationship to cerebral spinal fluid pressure. J. Nerv. Ment. Dis. 74: 192, 1931.

12. Osterholm, J. L. Reaction of the cerebral venous sinus system to acute intracranial hypertension. J. Neurosurg. 32: 654, 1970.

13. Queckenstedt, H. Zur Diagnose der Ruckenmarkkompression. Deut. Z. Nervenheilkd. 55: 325, 1916.

14. Shulman, K. Small artery and vein pressures in the subarachnoid space of the dog. J. Surg. Res. 5: $56,1965$.

15. Weed, L. H., and Flexner, L. B. The relations of the intracranial pressures. Amer. J. Physiol. 105: 266, 1933.

16. Wolff, H. G., and Forbes, H. S. The cercbral circulation: $\mathrm{V}$, Observations of the pial circulation during changes in intracranial pressure. Arch. Neurol. Psychiatr. (Chicago) 20: 1035, 1928.

17. Zwetnow, N. CBF autoregulation to blood pressure and intracranial pressure variations. Scand.J. Clin. Lab. Invest. Suppl. 102: 22, V-A, 1968. 